\title{
Éditorial
}

\section{Chers collègues}

Avec l'année 2019, vient l'abandon annoncé de la formule papier et le passage au tout-électronique et à l'open-access. Aujourd'hui, tous les articles du BSGF-Earth Sciences Bulletin sont accessibles gratuitement en ligne à tous les lecteurs, qu'ils soient ou non membres de la SGF. Cette évolution est une nécessité pour nous adapter à l'évolution du monde de la publication scientifique et à la demande des gouvernements européens qui considèrent à juste titre que la recherche payée par le contribuable doit être accessible librement. Nous sommes ici en avance sur nombre de nos concurrents qui devront suivre le même chemin à brève échéance. La somme que nous demandons aux auteurs pour cette nouvelle politique éditoriale est très modique par rapport à ce que demandent de grands concurrents (environ dix fois plus chers que nous). Nous avons donc aujourd'hui à notre disposition une revue française dont l'entièreté du processus éditorial, de la soumission des articles jusqu'à leur mise en ligne est totalement dématérialisée, induisant donc un raccourcissement considérable du délai de publication de nos recherches.

C'est maintenant à vous de faire vivre cet outil rénové. L'open-access total et la publication dans des délais très courts font qu'il n'y a plus aucune raison de ne pas se saisir de cette opportunité nouvelle et mettre les résultats de nos travaux à la disposition du plus grand nombre par le canal du BSGF-Earth Sciences Bulletin. Ce qui compte en définitive, ce n'est pas l'impact factor de la revue dans laquelle nous publions, mais bien l'impact de l'article que nous avons publié. Avec cette nouvelle formule, tous nos articles sont accessibles gratuitement dans le monde entier, bien plus efficacement qu'avec la formule traditionnelle, et nous devrions voir nos contributions plus souvent citées dans la littérature internationale. Pour ce faire, la majorité des articles que nous publions sont rédigés en anglais, mais il reste aux auteurs la possibilité de soumettre des contributions en français.

Mais cela demande que notre communauté s'empare du $B S G F$ et que chacun d'entre nous donne à la SGF une partie de ses résultats les plus marquants. Je m'adresse ici à l'ensemble de notre communauté et en particulier à ceux de nos collègues dont la notoriété internationale n'est plus à faire et dont les contributions au $B S G F$ aideraient à lui donner une plus grande visibilité.

Nous avons élargi la palette des spécialités de nos éditeursassociés afin que l'ensemble des spécialités des géologues (lato sensu) de France et du Monde puissent y trouver sa place. Le $B S G F$ publie tous les articles qui passent la barre de la revue par les pairs, quelle que soit la spécialité, de la géophysique et la géochimie profonde aux processus de surface et la paléontologie, ou les liens avec l'environnement. Les travaux de recherche fondamentale comme de recherche appliquée sont également bienvenus dans nos pages. Nous sommes totalement ouverts également aux articles traitant d'histoire des sciences de la Terre ou du patrimoine géologique. Les rapports de campagnes à la mer, d'acquisition de données géophysique, de nouvelles cartes géologiques, guides d'excursion, entre autres, peuvent également être pris en compte. En somme, toute la géologie, au sens général de l'étude de la Terre, a sa place dans le $B S G F$.

Pour favoriser le développement du $B S G F$, la SGF propose de financer la publication de trois nouveaux numéros spéciaux, avec la seule condition que les articles soient mis en ligne avant la fin de 2019. Si vous souhaitez profiter de cette opportunité, veuillez nous contacter avec un thème et une liste d'articles prévisionnelle.

Mes chers collègues, l'avenir du BSGF-Earth Sciences Bulletin est entre vos mains. Les outils pour publier rapidement en open-acces total pour un prix plus que raisonnable sont à votre disposition.

Avec les vœux de l'ensemble de la rédaction pour cette nouvelle année.

Laurent Jolivet

rédacteur en chef

laurent.jolivet@univ-orleans.fr 